\title{
Frequent Episodes of Brief Ischemia Sensitize the Fetal Sheep Brain to Neuronal Loss and Induce Striatal Injury
}

\author{
E. C. MAllard, C. E. Williams, A. J. GUNN, M. I. GUNNING, AND P. D. GLUCKMAN \\ Department of Pediatrics, University of Auckland, Auckland, New Zealand
}

\begin{abstract}
We examined the neuronal consequences of repeated brief episodes of in utero cerebral hypoxia-ischemia. Chronically instrumented fetal sheep were subjected to three 10 -min episodes of reversible cerebral ischemia, repeated at either 1-h $(n=8)$ or 5-h $(n=5)$ intervals. Four fetuses were subjected to a single 10 -min of ischemia and 17 fetuses to a single 30 -min of ischemia. Repeated insults altered the distribution of damage with relatively marked striatal injury compared with isolated episodes of ischemia $(p<0.01)$. Frequent insults were associated with greater neuronal loss $(p<0.01)$ and a failure to restore electrocorticographic activity and resolve cortical cytotoxic edema between insults. Intermittent cortical hyperexcitability developed after repeated insults irrespective of the interval between insults. These findings emphasize that repeated brief episodes of ischemia alter the distribution of damage and sensitize the fetal brain to neuronal injury, particularly if the episodes are frequent. Striatal damage may be a feature of multiple but not single insults. (Pediatr Res 33: 61-65, 1993)
\end{abstract}

\section{Abbreviations}

ECoG, electrocorticogram

$\mathrm{dB}$, decibels

Epidemiologic studies have often failed to find a clear association between isolated episodes of fetal asphyxia and neurologic outcome in the newborn $(1,2)$. This discrepancy may be due, in part, to multiple brief episodes of in utero asphyxia being unrecognized but more common than major and identifiable single events. However, few experimental studies have considered the neurologic consequences of multiple insults. Limited evidence from studies in the adult rat and gerbil suggests that brief episodes of ischemia repeated during the period of postischemic hypoperfusion may produce additive degrees of neuronal damage in the cortex, hippocampus, and caudate nucleus and vasogenic edema $(3,4)$. We have previously shown that the parasagittal cortex and hippocampus are particularly vulnerable to both brief $(10-\mathrm{min})$ and prolonged (30- to 40-min) single episodes of cerebral ischemia or global asphyxia in the fetal sheep $(5,6)$. However, despite frequent clinical observations of striatal damage with perinatal encephalopathy (7), neither cerebral ischemia nor global asphyxia induced predominantly striatal patterns of injury.

The objective of the study presented here was to determine whether multiple brief episodes of ischemia sensitize the fetal

Received May 26, 1992; accepted August 31, 1992.

Correspondence: Professor Peter D. Gluckman, Department of Pediatrics, University of Auckland, Private Bag, Auckland, New Zealand.

Supported by a program grant from the Health Research Council of New Zealand. brain to injury or alter the pattern of damage compared with isolated insults and whether the length of the interval between insults is of importance. We have previously described an experimental preparation of transient cerebral ischemia in the chronically instrumented fetal sheep that induces encephalopathy similar to hypoxic-ischemic brain damage observed in some asphyxiated term infants (8). Therefore, we investigated the electrophysiologic and histologic consequences of isolated and brief episodes of reversible cerebral ischemia repeated at either I or $5 \mathrm{~h}$. Fetal peripheral lactate and glucose levels were measured. We demonstrate that repeated insults, particularly if frequent, are associated with an altered distribution of neuronal loss compared to isolated insults, the striatum being particularly sensitive. The frequency of insults appear to have a major effect on the severity of outcome.

\section{MATERIALS AND METHODS}

These studies were approved by the Animal Ethical Committee of the University of Auckland. Thirty-four pregnant ewes with fetuses at 119-127 d of gestation underwent operation under $2 \%$ halothane/oxygen general anesthesia as described previously (8). Briefly, polyvinyl catheters were placed in each fetal axillary artery and in the amniotic cavity. Through burr holes, two pairs of ECoG electrodes (5 and $15 \mathrm{~mm}$ anterior and $10 \mathrm{~mm}$ lateral to bregma) and one pair of stimulating electrodes to measure cortical impedance $(10 \mathrm{~mm}$ anterior and $15 \mathrm{~mm}$ lateral of bregma) were placed extradurally over the parasagittal cortex. To restrict cerebral vascular supply to the carotid arteries only, the vertebro-occipital anastomoses between the carotid arteries and vertebral arteries were ligated bilaterally. Two pairs of inflatable cuffs were placed around the carotid arteries.

Studies commenced $3 \mathrm{~d}$ after surgery. Fetal ECoG and impedance signals were recorded as previously described (5). Briefly, ECoG spectrum and cortical impedance were collected in real time for $12 \mathrm{~h}$ before occlusion and for $72 \mathrm{~h}$ afterward. Cortical impedance measurements can be used to estimate the changes in extracellular space that occur concomitantly with cytotoxic edema (9). Reversible cerebral ischemia was achieved by inflating the carotid cuffs bilaterally with sterile saline for a defined period and then reversing by deflation. Fetal blood samples were drawn immediately before and after each occlusion and at postmortem and analyzed for $\mathrm{pH}$, oxygen content, $\mathrm{PCO}_{2}$, lactate, and glucose.

Four protocols were followed. In the first protocol, three 10min carotid artery occlusions were performed at $1-\mathrm{h}$ intervals $(3$ $\times 10 \mathrm{~min}, 1 \mathrm{~h}$ ), whereas in the second protocol, three 10 -min occlusions were repeated at 5 -h intervals $(3 \times 10 \mathrm{~min}, 5 \mathrm{~h})$. Fetuses were randomly assigned to either protocol. In the third protocol, a single 10-min episode of ischemia was induced, and in the fourth protocol, a single 30 -min episode of ischemia was induced. Data from the single 10-min and 30-min occlusions have been reported elsewhere (5). In the present study, only histologic outcome from these latter fetuses was considered, as 
the configuration of electronic processing units previously used does not allow a valid direct comparison. Eight fetuses underwent the first protocol $(3 \times 10 \mathrm{~min}), 1 \mathrm{~h}$, three of which died $60-65 \mathrm{~h}$ after the last occlusion due to proven infection. These three fetuses were excluded from final ECoG measurements and histologic analysis. Five other fetuses were subjected to the second protocol $(3 \times 10 \mathrm{~min}, 5 \mathrm{~h})$, four fetuses to a single 10 -min episode, and 17 fetuses to a single 30 -min episode of ischemia.

Three $d$ after the last occlusion, fetuses were killed by an overdose of sodium pentobarbitone, and the fetal brain was prepared for histologic analysis as previously described (5). Briefly, the brain was perfused in situ with formaldehyde, acetic acid, and methanol (1:1:8), paraffin-embedded, and sectioned at $8 \mu \mathrm{m}$. Histologic slides were stained with thionin and acid fuchsin.

Analysis. ECoG intensity was calculated as previously described and normalized with respect to the 12-h reference period (10). Suppression of ECoG intensity postinsult was defined as activity below $-5 \mathrm{~dB}$ relative to the reference period. Final intensity measurements were based on the last $4 \mathrm{~h}$ of the experiment. Hyperexcitability was defined as intensity at least $5 \mathrm{~dB}$ above baseline. Cortical impedance was extracted from the ECoG signal, and estimated changes in extracellular space were derived from these measurements as previously described (9). Impedance was normalized and expressed as a percentage of baseline.

Neuronal loss was determined under light microscopy by two independent assessors, one of whom was blind to the experiment. The correlation of neuronal loss scores between the two assessors was $r^{2}=0.92$. Neurons showing ischemic cell change with acidophilic (red) cytoplasm and contracted nuclei were considered dead (5). Damage scores from predetermined regions were as follows: $0=$ no dead neurons, $5=1-10 \%, 30=11-50 \%, 70$ $=51-90 \%, 95=91-99 \%$, and $100=100 \%$ dead neurons.

Histologic scores, cortical extracellular space, lactate and glucose measurements, and final 4-h ECoG recordings were subjected to analysis of variance. Region and time were treated as repeated measures as appropriate. When overall differences were found, between-group comparisons were made using the Newman-Keul's multiple comparison test. All data are presented as mean \pm SEM.

\section{RESULTS}

$3 \times 10$ Min ischemia separated by $1 \mathrm{~h}$. During each occlusion, the ECoG became isoelectric, and ECOG activity remained suppressed between occlusions (Fig. 1). ECoG suppression lasted for $5 \pm 0.7 \mathrm{~h}$ after the initial occlusion. Hyperexcitability developed $9 \pm 3 \mathrm{~h}$ after the initial occlusion and lasted for $29 \pm 8 \mathrm{~h}$. Two animals developed continuous seizure activity, whereas the other six showed discrete episodes of hyperexcitability. By $3 \mathrm{~d}$, ECoG intensity was $-4 \pm 2 \mathrm{~dB}$, which was not different from baseline.

Cortical impedance increased during each occlusion (Fig. 2). After recirculation, the impedance recovered toward preinsult values but did not fully resolve before the second and third occlusion. Thus, there was an increase in impedance with time, each occlusion resulting in a further increase, which was also reflected in loss of estimated extracellular space $(p<0.01$, Fig. $3)$. Cortical impedance returned to baseline after the third occlusion, after which there were no changes except in the two animals with continuous seizure activity that was associated with a secondary rise in impedance.

A progressive metabolic acidosis developed with the occlusions (Table 1). Glucose levels increased after the first occlusion and then stabilized at that level during the second and third occlusions (Table 2). No changes were observed in oxygen content or $\mathrm{PCO}_{2}$ during the experiment.

The distribution of neuronal loss is shown in Figure 4. Overall neuronal loss was similar to that in fetuses subjected to a single 30 -min episode of ischemia $(p=0.39)$, but the distribution of damage differed ( $p<0.001$, Fig. 5). Striatal damage was signifi-
EEG intensity $(\mathrm{dB})$
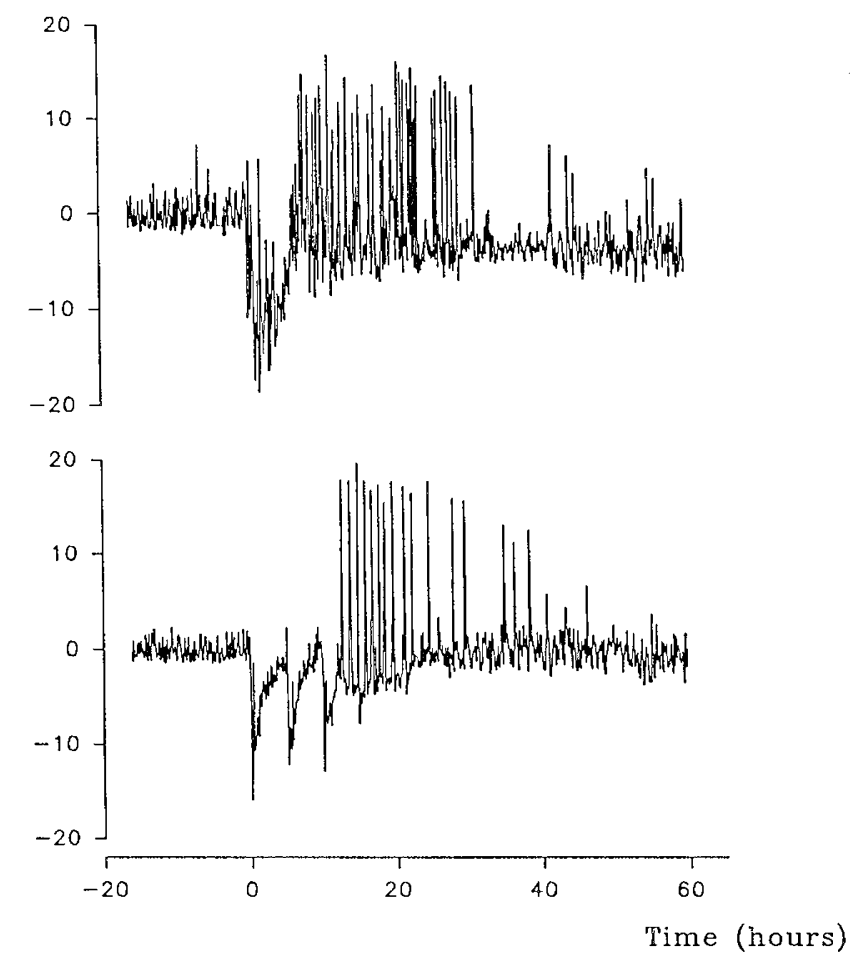

Fig. 1. Typical examples of the ECoG intensity changes during and after $3 \times 10 \mathrm{~min}$ of ischemia repeated every $1 \mathrm{~h}$ (upper panel) and every $5 \mathrm{~h}$ (lower panel). The first occlusion is at time zero in both experiments.

cantly increased $(p<0.01)$ and parasagittal cortical damage was significantly decreased $(p<0.01)$ compared with a single $30-$ min episode of ischemia.

$3 \times 10 \mathrm{Min}$ ischemia separated by $5 \mathrm{~h}$. After an isoelectric ECoG during each occlusion, ECoG returned to baseline between insults (Fig. 1). In four of five animals, discrete episodes of hyperexcitability, similar to those in animals subjected to the 3 $\times 10 \mathrm{~min}, 1 \mathrm{~h}$ protocol, developed $8 \pm 2 \mathrm{~h}$ after the first occlusion with a duration of $44 \pm 9 \mathrm{~h}$. Final ECoG activity $(-0.3 \pm 1 \mathrm{~dB})$ was not different from baseline or that in the $3 \times 10 \mathrm{~min}, 1 \mathrm{~h}$ protocol.

There was an increase in cortical impedance during each occlusion, with all three insults resulting in similar increases (Fig. 2). After the third occlusion, cortical impedance returned to baseline, and there was no change in impedance throughout the rest of the experiment. No changes in metabolic parameters were observed (Tables 1, 2).

Overall neuronal loss was slight (Fig. 4) and was similar to that resulting from a single 10 -min episode of ischemia $(p=0.48$, Fig. 5), except for marked striatal damage. Neuronal loss after the $3 \times 10 \mathrm{~min}, 5 \mathrm{~h}$ protocol was less severe than that after the $3 \times 10,1$ h protocol $(p<0.01)$ but showed a similar distribution, with striatal damage being predominant in both protocols.

$1 \times 10 \mathrm{~min}$ ischemia. The electrophysiologic and histologic data are reported in detail elsewhere (5). The distribution of neuronal loss is shown in Figure 5. No postinsult hyperexcitability or prolonged disturbances in cortical impedance or ECoG intensity were observed.

$1 \times 30 \mathrm{~min}$ ischemia. The electrophysiologic and histologic data are reported elsewhere (5). A period of postischemic continuous seizure activity associated with a secondary rise in cortical impedance was observed. We have previously reported a loss of ECoG intensity of $-10 \pm 5 \mathrm{~dB} 3 \mathrm{~d}$ after injury (5). The distribution of neuronal loss is shown in Figure 5. 
Cortical Impedance (\%)
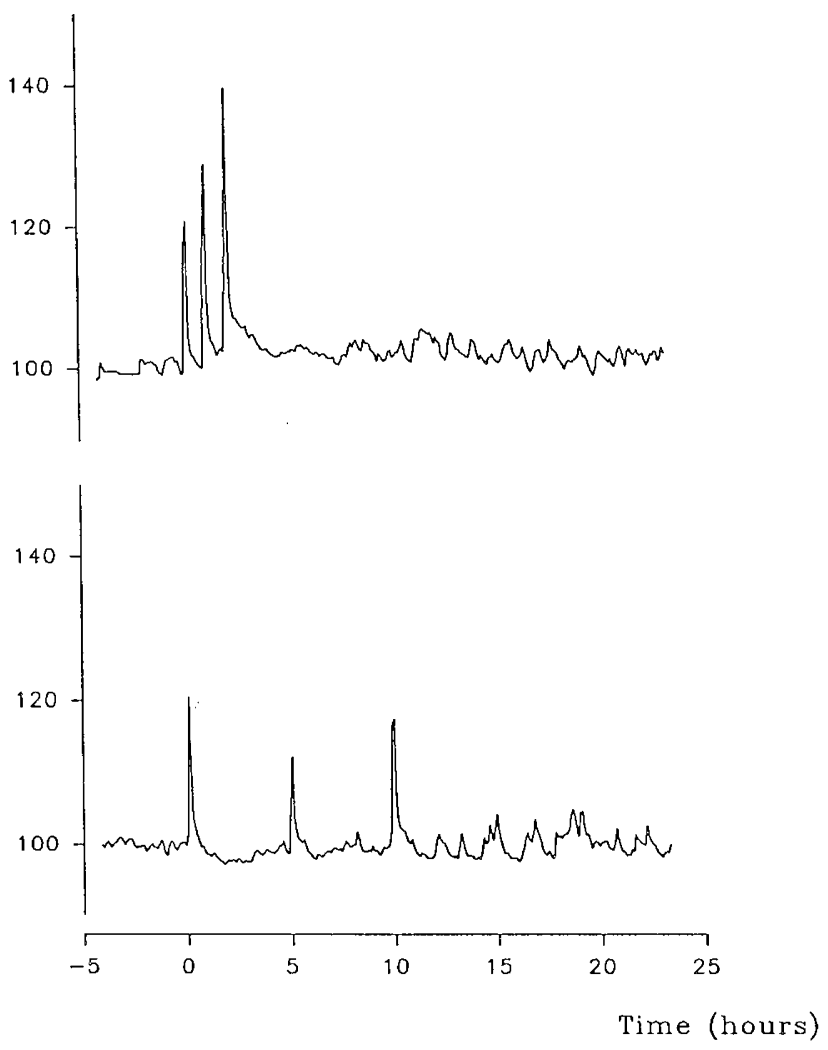

Fig. 2. Changes in cortical impedance relative to baseline (100\%) after $3 \times 10 \mathrm{~min}$ of transient cerebral ischemia repeated at 1-h (upper panel) and 5-h (lower panel) intervals. Time zero indicates the first occlusion.
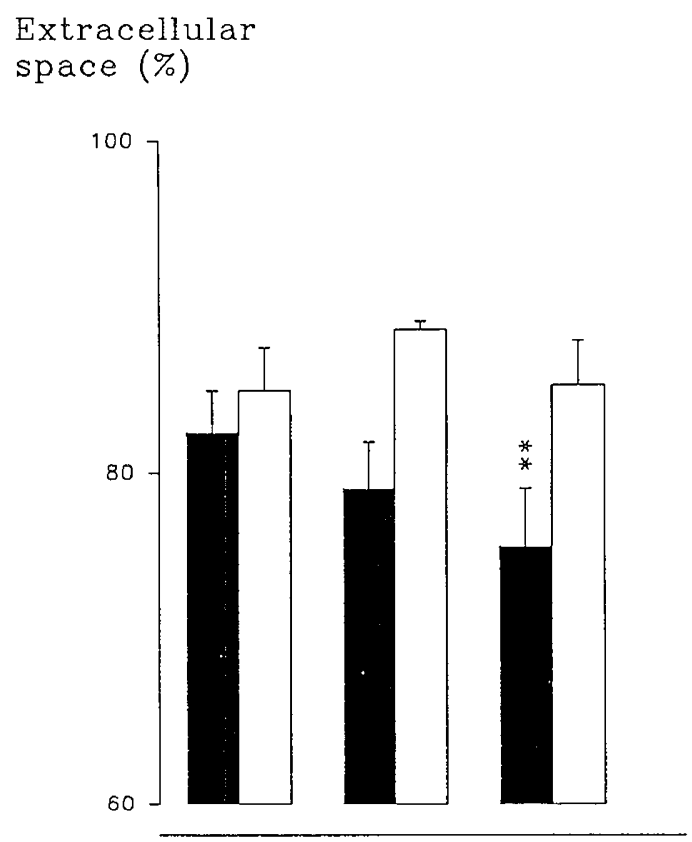

Insult 1 Insult 2 Insult 3

Fig. 3. Maximum decrease of estimated extracellular space during each occlusion relative to baseline $(100 \%)$. Changes in estimated extracellular space are derived from cortical impedance measurements. $\mathbf{\square}, 3$ $\times 10$-min insults, $1 \mathrm{~h}$ apart; $\square, 3 \times 10$-min insults, $5 \mathrm{~h}$ apart. ${ }^{* *}, p<$ 0.01 compared with the first insult.
Table 1. Fetal arterial lactate levels $(\mathrm{mmol} / \mathrm{L})$

\begin{tabular}{llll}
\hline & Preinsult 1 & Preinsult 2 & Preinsult 3 \\
\hline $3 \times 10 \mathrm{~min}$, 1 h apart & $0.9 \pm 0.06$ & $2.0 \pm 0.2^{*}$ & $2.6 \pm 0.4^{*}$ \\
$3 \times 10 \mathrm{~min}, 5$ h apart & $0.8 \pm 0.09$ & $0.9 \pm 0.2$ & $1.2 \pm 0.2$ \\
\hline
\end{tabular}

$* p<0.01$ compared with the first insult.

Table 2. Fetal arterial glucose levels ( $\mathrm{mmol} / \mathrm{L}$ )

\begin{tabular}{cccl}
\hline & Preinsult 1 & Preinsult 2 & Preinsult 3 \\
\hline $3 \times 10 \mathrm{~min}$, 1 h apart & $1.0 \pm 0.09$ & $1.5 \pm 0.1^{*}$ & $1.5 \pm 0.1^{*}$ \\
$3 \times 10 \mathrm{~min}$, 5 h, apart & $0.9 \pm 0.07$ & $1.0 \pm 0.1$ & $1.2 \pm 0.1$ \\
\hline
\end{tabular}

${ }^{*} p<0.01$ compared with the first insult.

Region
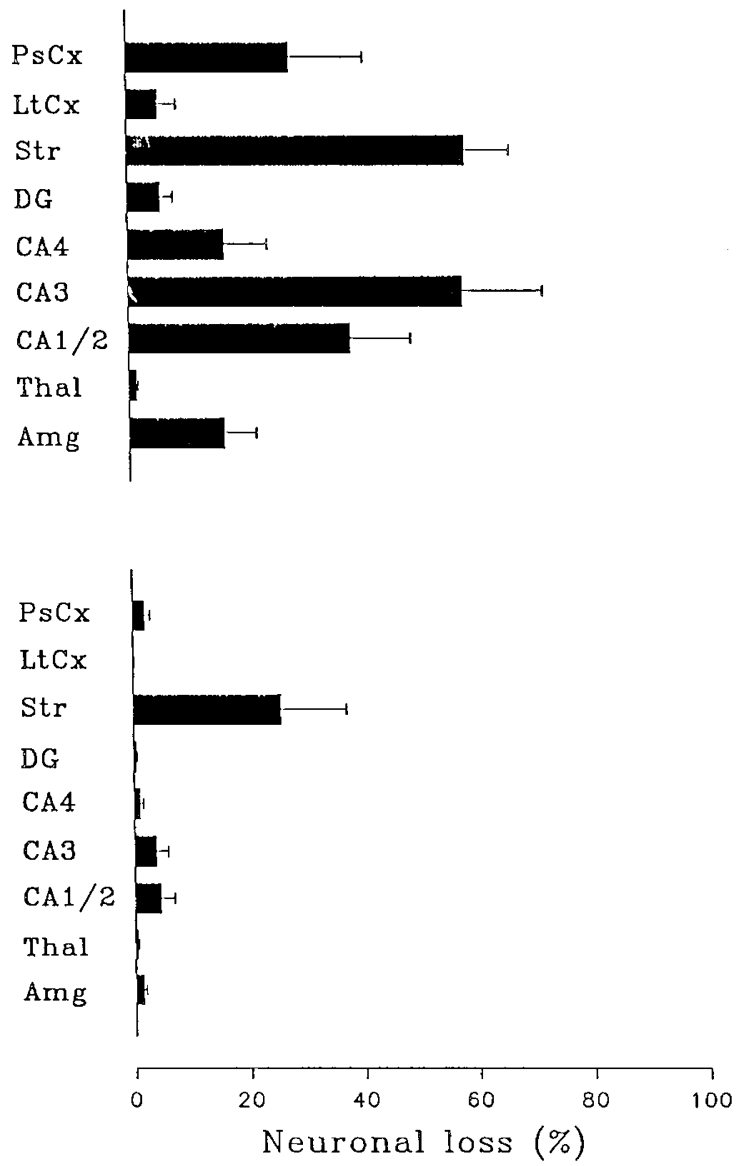

Fig. 4. Distribution of histologic damage in fetuses subjected to $3 \times$ 10-min insults at 1 -h (upper panel) or 5-h (lower panel) intervals. Animals were killed $72 \mathrm{~h}$ after the last insult. PsCx, Parasagittal cortex; $L t C x$, lateral cortex; Str, striatum; $D G$, dentate gyrus; $C A 4$, CA4 region of the hippocampus; $C A 3, \mathrm{CA} 3$ region of the hippocampus; $C A 1 / 2, \mathrm{CA} 1$ and $\mathrm{CA} 2$ regions of the hippocampus; Thal, thalamus; and $A m g$, amygdala.

\section{DISCUSSION}

Choreoathetosis secondary to striatal injury is one of the classic syndromes of cerebral palsy and is presumed to be a result of striatal injury in the perinatal period (7). However, despite its clinical importance, little is known about the etiology of such perinatal injury (11). Attempts to correlate pregnancy risk factors with the various patterns of cerebral palsy have not been successful (12). Similarly, attempts to relate immediate perinatal events to the different clinical outcomes have not been elucidatory.

After repeated short episodes of ischemia, irrespective of the 


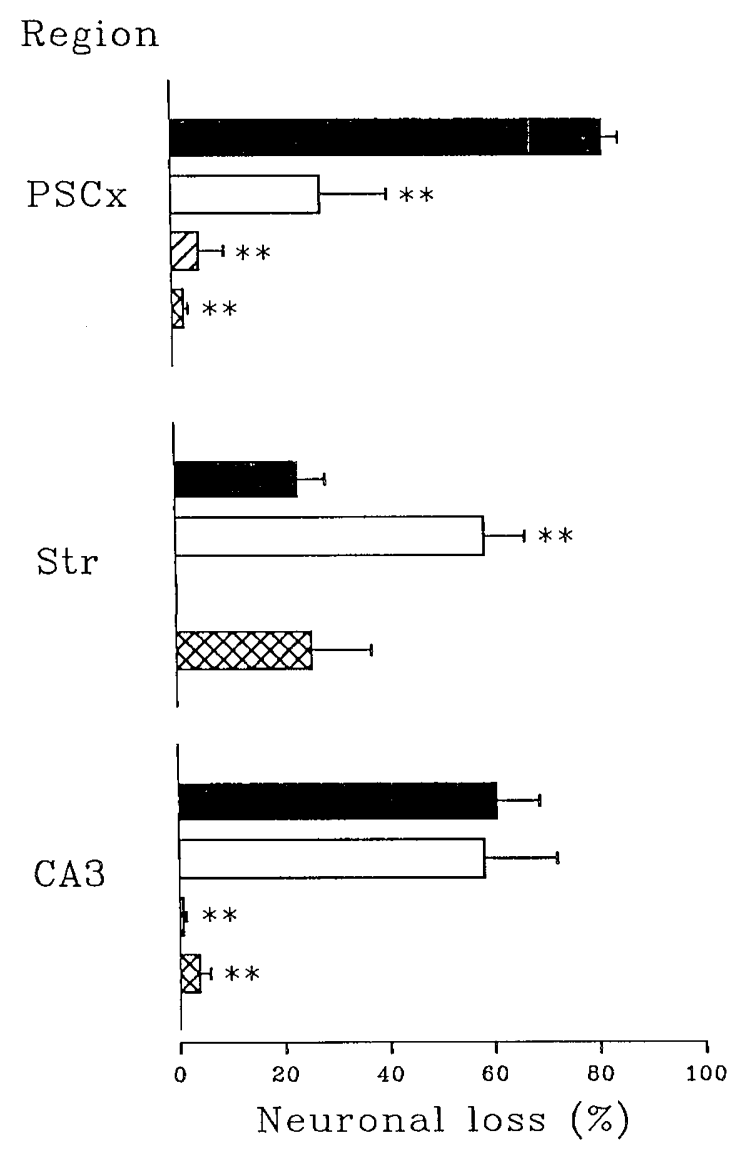

Fig. 5. Histologic outcome in selective regions $3 \mathrm{~d}$ after transient cerebral ischemia. PsCx, Parasagittal cortex; Str, striatum; and $C A 3, \mathrm{CA} 3$ region of the hippocampus. $\mathbf{D}, 1 \times 30 \mathrm{~min}, \square, 3 \times 10 \mathrm{~min}, 1 \mathrm{~h}$ apart; $\mathbb{Z}$, $1 \times 10 \mathrm{~min} ; \otimes, 3 \times 10 \mathrm{~min}, 5 \mathrm{~h}$ apart. ${ }^{* *}, p<0.01$ compared with a single 30 -min episode of ischemia.

interval between insults, striatal damage was a dominant histologic finding. In contrast with these results, we have previously shown that a single 10-min episode of ischemia in the fetal sheep causes trivial cerebral damage, whereas an isolated, more prolonged episode of ischemia (30 min) results in severe neuronal loss in the hippocampus and infarction of the parasagittal cortex but little damage in the striatum $(5,13)$. Although these previous studies used the same protocol, it is possible that conditions were not identical, which may have influenced the outcome. In other experimental approaches to isolated episodes of in utero asphyxia, such as umbilical cord occlusion, we have demonstrated particular vulnerability of the hippocampus (6). Therefore, it appears that the distribution of damage may be dependent on the temporal sequencing as well as the specific nature of the insult. Marked striatal damage may be a feature of multiple, but not isolated, in utero insults.

$\dot{A}$ number of mechanisms have been implicated in hypoxicischemia cell loss, including intracellular sodium and water accumulation leading to lysis, intracellular calcium accumulation, and toxic effects of excitatory amino acids (14). Injury to hippocampal neurons after ischemic insults has been associated with excessive release of glutamate (15). However, whether similar mechanisms apply to striatal damage after ischemia is not clear. In developing rats, the striatum and hippocampus contain a high density of postsynaptic glutamate receptors (16). Immediately after severe global asphyxia in exteriorized fetal sheep, the concentration of extracellular excitatory amino acids increases markedly, particularly in the striatum (17). Interactions between corticostriatal glutamateric pathways and nigrostriatal dopaminergic afferents have also been suggested to play a role in postischemic striatal damage (18). Creating lesions in the substantial nigra prevented the marked postasphyxial increase in striatal dopamine and attenuated striatal neuronal loss (19). Striatal neurons, particularly small- to medium-sized ones, have been shown to attain ischemic cell change more rapidly than cortical and hippocampal neurons (20). This early degeneration of small- to medium-sized striatal neurons has been related to changes in striatal dopamine neurohistochemistry (21). It may be that repeated insults enhance these early ischemic cell changes, sensitizing striatal neurons in particular.

The magnitude of neuronal loss after multiple episodes of ischemia in the present study was related to the interval between occlusions, cerebral damage being greater after more frequent insults. Three 10 -min insults repeated at 1 -h intervals showed an amount of overall damage similar to that in a single 30 -min ischemic insult, whereas three 10 -min insults $5 \mathrm{~h}$ apart resembled a single 10-min ischemic insult. These results are similar to those in studies of adult rats demonstrating more damage in the hippocampus after three 3-min four-vessel occlusions delivered hourly than after a single 9-min occlusion (4). Similarly, repeated cerebral ischemia in the adult gerbil caused greater neuronal damage after three 5-min episodes of ischemia at 1 -h intervals than $15 \mathrm{~min}$ of ischemia produced as a single insult (3). The increased damage in those experiments was suggested to result from repeated insults coinciding with the time of postischemic cerebral hypoperfusion (3), although other mechanisms must be considered. For example, the cytotoxic milieu, as a result of free radical production, excitatory amino acid and $\mathrm{Ca}^{2+}$ release, and changes in extracellular volume may place the neuron in a more vulnerable state when episodes of ischemia are repeated more frequently. Conversely, homeostatic mechanisms, including the release of inhibitory neuromodulators such as adenosine and the gradual resolution of cytotoxic accumulation, may mean that greater protection is offered by less frequent insults. Indeed, infrequent episodes of ischemia in the adult gerbil have been shown to be neuroprotective (22).

Postischemic hypoperfusion has been related to cerebral edema in the adult rat (23), and vasogenic edema was found $24 \mathrm{~h}$ after ischemic insults repeated at $1-\mathrm{h}$ intervals in gerbils (3). However, these studies did not measure the acute cytotoxic edema formation during insults. In the fetal sheep, we have previously reported transient loss of extracellular space due to cytotoxic edema during ischemic insults (9). The intracellular swelling is understood to result from increased $\mathrm{Na}^{+}$and $\mathrm{Cl}^{-}$influx and uptake of osmotic water (24). In vitro studies of hippocampal cells have suggested that cellular swelling plays a role in neuronal death (25). Our data show that frequently repeated insults lead to progressively worse cytotoxic edema during the insult period. The exact neuronal consequences of this phenomenon are unclear but are likely to reflect longer-lasting defects in cellular integrity. This incomplete restoration of membrane function could leave the neuron more sensitive to the ion and water fluxes associated with the frequently repeated insults.

We found that there was a prolonged depression of ECoG activity after occlusions at $1-\mathrm{h}$ intervals, whereas ECoG activity recovered between insults when they were separated by $5 \mathrm{~h}$. After isolated transient ischemic insults in the fetal sheep, prolonged ECoG depression is associated with cortical injury (5). Although the underlying mechanisms are not well understood, depression of ECoG activity reflects loss of electrical activity. Electrical activity and trophic factors such as nerve growth factor and IL1 have been suggested to regulate neuronal survival in vitro $(26$, 27). We have recently shown that other neurotropic factors such as IGF-I are induced after hypoxic-ischemic injury in the rat brain and that postasphyxial central administration of IGF-I is neuroprotective (28). Possibly, neuronal loss in the present study was diminished after less frequent insults because the ECoG activity recovered between insults, allowing for induction of neurotrophic action.

We have previously shown that continuous seizure activity after $30 \mathrm{~min}$ of ischemia in the fetal sheep is associated with 
infarction of the underlying parasagittal cortex (8) and that intermittent hyperexcitability develops after uterine artery occlusion only when mild to moderate neuronal loss is found in the parasagittal cortex (29). However, suppression of these seizures with an N-methyl-D-aspartate receptor antagonist, MK-801, improves neuronal outcome in the lateral cortex and the hippocampus but not in the parasagittal cortex (13). Similarly, in this study, discrete episodes of hyperexcitability were associated with mild to moderate degrees of cortical neuronal loss after repeated insults.

Metabolic factors have long been considered to have some effect on outcome of cerebral hypoxic-ischemic insults. Systemic hyperglycemia before ischemia is known to worsen outcome in adult rats (30) but may be neuroprotective in immature rats (31). Similarly, elevated systemic lactate has long been thought to play a role in the development of ischemic brain damage in monkeys (32). We observed increased plasma lactate and glucose levels after the initial occlusion, and these changes were more pronounced in the protocol with 1 -h intervals. However, the peripheral acidosis and hyperglycemia observed and presumed to reflect cerebral concentrations were only mild, and it is doubtful that such small changes could have a central role in the differences in outcome found in this study.

The present study shows that brief episodes of ischemia, repeated before full recovery of ECoG and intracellular edema, sensitize neurons to damage. Repeated insults, independent of the interval between the insults, alter the pattern of brain injury. This implies that the pattern rather than the nature of the insult may be important in certain neuropathologies. In particular, striatal damage appears to be a feature of multiple but not single insults. These observations offer at least a partial explanation of epidemiologic difficulties linking perinatal events with subsequent outcome.

Acknowledgment. The authors thank Dr. B. M. Johnston for her helpful advice.

\section{REFERENCES}

1. Mann LI 1986 Pregnancy events and brain damage. Am J Obstet Gynecol 155:6-9

2. Paneth N, Stark RI 1983 Cerebral palsy and mental retardation in relation to indicators of perinatal asphyxia. Am J Obstet Gynecol 147:960-966

3. Tomida S, Nowak TS, Vass K, Lohr JM, Klatzo I 1987 Experimental model for repetitive attacks in the gerbil: the cumulative effect of repeated ischemic insults. J Cereb Blood Flow Metab 7:773-782

4. Nakano S, Kato H, Kogure K 1989 Neuronal damage in the rat hippocampus in a new model of repeated reversible transient cerebral ischemia. Brain Res 490:178-180

5. Williams CE, Gunn AJ, Mallard EC, Gluckman PD 1992 Outcome after ischemia in the developing sheep brain: an electroencephalographic and histological study. Ann Neurol 31:14-21

6. Mallard EC, Gunn AJ, Williams CE, Gluckman PD 1992 Transient umbilical cord occlusion causes hippocampal damage in fetal sheep. Am J Obstet Gynecol (in press)

7. Spiegel EA, Baird HW 1968 Athetotic syndromes. In: Vinken PJ, Bruyn GW (eds) Handbook of Clinical Neurology. Diseases of the Basal Ganglia. North Holland, Amsterdam, pp 440-475

8. Williams CE, Gunn AJ, Gluckman PD, Synek B 1990 Delayed seizures occurring with hypoxic-ischemic encephalopathy in the fetal sheep. Pediatr Res 27:561-565
9. Williams CE Gunn AJ, Gluckman PD 1991 Time course of intracellular edema and epileptiform activity following prenatal cerebral ischemia in sheep. Stroke 22:516-521

10. Williams CE, Gluckman PD 1990 Real-time spectral intensity analysis of the EEG on a microcomputer. J Neurosci Methods 32:9-13

11. Stanley FJ 1984 Prenatal risk factors in the study of the cerebral palsies. In: Stanley FJ, Alberman ED (eds) The Epidemiology of the Cerebral Palsies. Spastics International Medical Publications, London, pp 87-97

12. Nelson KB, Ellenberg JH 1986 Antecedents of cerebral palsy: multivariate analysis of risk. $\mathrm{N}$ Engl $\mathrm{J}$ Med 315:81-86

13. Tan KMW, Williams CE, Gunn AJ, Mallard EC, Gluckman PD 1992 Suppression of post-ischemic epileptiform activity with MK-801 improves neural outcome in fetal sheep. Ann Neurol (in press)

14. Williams CE, Gluckman PD, Gunn AJ, Tan WK, Dragunow M 1990 Perinatal asphyxic brain damage: the potential for therapeutic intervention. In: Dawes GS, Zacutti A, Borruto F, Zacutti Jr A (eds) Fetal Autonomy and Adaptation. John Wiley, New York, pp 145-163

15. Choi DW 1990 The role of glutamate neurotoxicity in hypoxic-ischemic neuronal death. Annu Rev Neurosci 13:171-182

16. Silverstein FS, Chen R, Johnston MV 1986 The glutamate analogue quisqualic acid is neurotoxic in striatum and hippocampus of immature rat brain. Neurosci Lett 71:13-18

17. Hagberg H, Andersson P, Kjellmer I, Thiringer K, Thordstein M 1987 Extracellular overflow of glutamate, aspartate, GABA, and taurine in the cortex and basal ganglia of fetal lambs during hypoxia-ischemia. Neurosci Lett $78: 311-317$

18. Globus MY, Busto R, Dietrich WD, Martinez E, Valdes I, Ginsberg MD 1988 Effect of ischemia on the in vivo release of striatal dopamine, glutamate and $\gamma$-aminobutyric acid studied by intracerebral microdialysis. J Neurochem 51:1455-1464

19. Globus MY, Ginsberg MD, Dietrich WD, Busto R, Scheinberg P 1987 Substantia nigra lesion protects against ischemic damage in the striatum. Neurosci Lett 80:251-256

20. Pulsinelli W, Brierley JB, Plum F 1982 Temporal profile of neuronal damage in a model of transient forebrain ischemia. Ann Neurol 11:491-498

21. Nemeth G, Cintra A, Herb J-M, Ding A, Goldstein M, Agnati LF, Hoyer S, Fuxe K 1991 Changes in striatal dopamine neurohistochemistry and biochemistry after incomplete transient cerebral ischemia in the rat. Exp Brain Res 86:545-554

22. Kirino T, Tsujita Y, Tamura A 1991 Induced tolerance to ischemia in gerbil hippocampal neurons. J Cereb Blood Flow Metab 11:299-307

23. Schurer L, Grogaard B, Arfors K-E, Gerdin B 1990 Is postischemic hypoperfusion related to brain edema? In: Long D (ed) Advances in Neurology, Vol 52. Raven Press, New York, pp 155-164

24. Randall JB, Hoff JT 1990 Ischemic cerebral edema. In: Weinstein PR, Faden AI (eds) Protection of the Brain from Ischemia. Williams \& Wilkins, Baltimore, pp 67-74

25. Rothman S 1985 The neurotoxicity of excitatory amino acids is produced by passive chloride influx. J Neurosci 5:1483-1489

26. Koike $\mathrm{T}$, Martin D, Johnson E 1989 Role of $\mathrm{Ca}^{2+}$ channels in the ability of membrane depolarization to prevent neuronal death induced by trophic factor deprivation: evidence that levels of internal $\mathrm{Ca}^{2+}$ determine NGF dependence of sympathetic ganglion cells. Proc Natl Acad Sci USA 86:64216425

27. Brenneman D, Schultzberg M, Bartfal T, Gozes I 1992 Cytokine regulation of neuronal survival. J Neurochem 2:454-460

28. Gluckman PD, Klempt ND, Guan J, Mallard EC, Sirimanne E, Dragunow M, Singh K, Klempt M, Williams CE, Nikolics K 1992 A role for IGF-I in the rescue of CNS neurons following hypoxic-ischemic injury. Biochem Biophys Res Commun 182:593-599

29. Gunn AJ, Parer JT, Mallard EC, Williams CE, Gluckman PD 1992 Cerebral histological and electrophysiological changes after asphyxia in fetal sheep. Pediatr Res 31:486-491

30. Warner D, Smith M, Siesjo B 1987 Ischemia in normo- and hyperglycemic rats: effects on brain water and electrolytes. Stroke 18:464-471

31. Voorhies TM, Rawlinson D, Vannucci RC 1986 Glucose and perinatal hypoxic-ischemic brain damage in the rat. Neurology $36: 1115-1118$

32. Yamaguchi M, Myers RE 1976 Comparison of brain biochemical changes produced by anoxia and hypoxia. J Neuropathol Exp Neurol 35:302 